\title{
Image Segmentation Techniques with Machine Learning
}

\author{
Raksha S Kale" ${ }^{* 1}$ Dr. Surabhi Thorat ${ }^{2}$ \\ ${ }^{*}$ Masters in Computer Science, Somaiya University, Mumbai, Maharashtra, India \\ ${ }^{2}$ Department of Computer Science, Somaiya University, Mumbai, Maharashtra, India
}

\section{Article Info}

Volume 7, Issue 6

Page Number: 232-235

\section{Publication Issue :}

November-December-2021

\section{Article History}

Accepted : 02 Dec 2021

Published : 10 Dec 2021

\section{ABSTRACT}

Image segmentation is the fundamental step to analyze images and extract data from them. This paper concentrates on the idea behind the basic methods used. Image segmentation is the process of partitioning a digital image into multiple segments(i. e. sets of pixels). The goal of segmentation is to simplify or change the representation of an image into something that is more meaningful and easier to analyze. Segmentation is typically used to locate objects and boundaries(line, curve) in image. The Image segmentation is a set of segments that collectively cover the entire image. Each of pixels in a region are similar with respect to some characteristics colour, intensity or texture. The various segmentation techniques discussed in this paper.

Keywords: Segmentation, Edge detection, Thresholding, Clustering, Region Based.

\section{INTRODUCTION}

Image processing is one of the most important techniques which is used in many areas like Object detection, Diagnostic, Image analysis, Surgical Planning Robotics, Localization of tumors, Trace objects in satellite images (roads, forests, etc. ), Traffic control systems, face recognition, Image Understanding Model, etc.

Image Segmentation is the process of assigning a label to every pixel in an image. Is a sub field of Artificial intelligence and computer vision. It is typically used to locate objects and boundaries in images.

We might have wondered how fast and efficiently our brain is trained to identify and classify what our eyes perceive. Somehow our brain is trained in a way to analyze everything at a granular level. This helps us distinguish an apple from a bunch of oranges.
Computer vision is a field of computer science that enables computers to identify and process objects in videos and images just the way we humans do.

\section{A. Object detection and image localization:}

When there is a single object present in an image, we use image localization technique to draw a bounding box around that object. In the case of object detection, it provides labels along with the bounding boxes; hence we can predict the location as well as the class to which each object belongs.

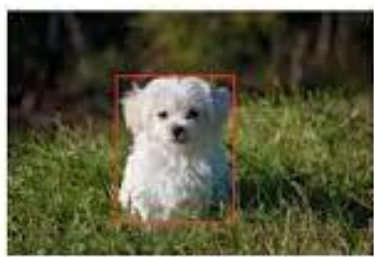

Image Localization

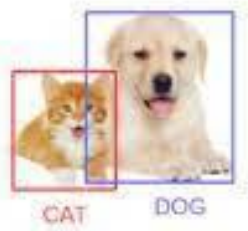

Object Detection 


\section{B. Types of image segmentation methods:}

\section{Semantic Segmentation :}

Semantic Segmentation is the process of segmenting the image pixels into their respective classes and is given the same label/color value.

\section{Instance Segmentation :}

Instance segmentation is more thorough and usually comes into picture when dealing with multiple objects. Every pixel of each object of a class is given a separate label/color value.

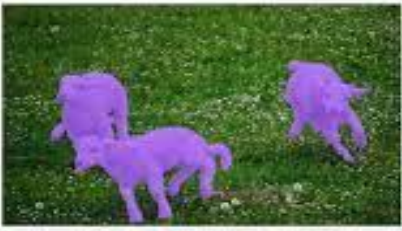

Serrontic segmentation

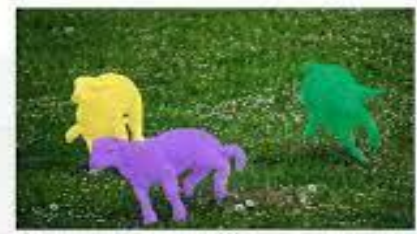

instance segmentotion

\section{METHODS AND MATERIAL}

\section{Segmentation by Edge Detection :}

In the image segmentation process, the basic step is edge detection. It divides an image into an object and its background. Edge detection divides the image by observing the change in intensity or pixels of an image.
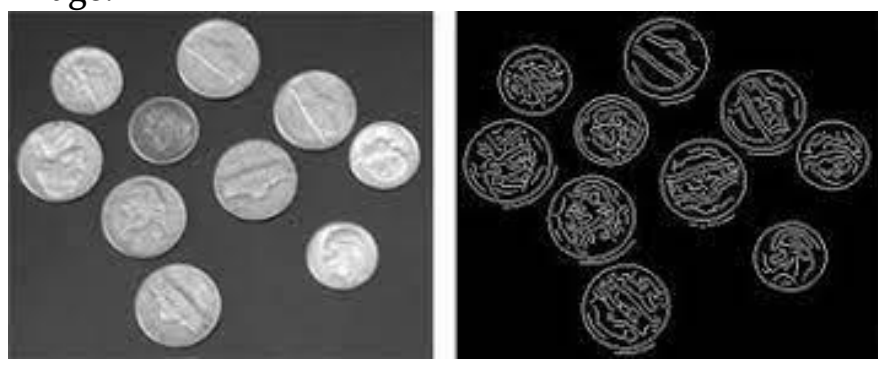

\section{Segmentation by Thresholding :}

One of the simplest approaches to segment an image is based on the intensity levels and is called as threshold based

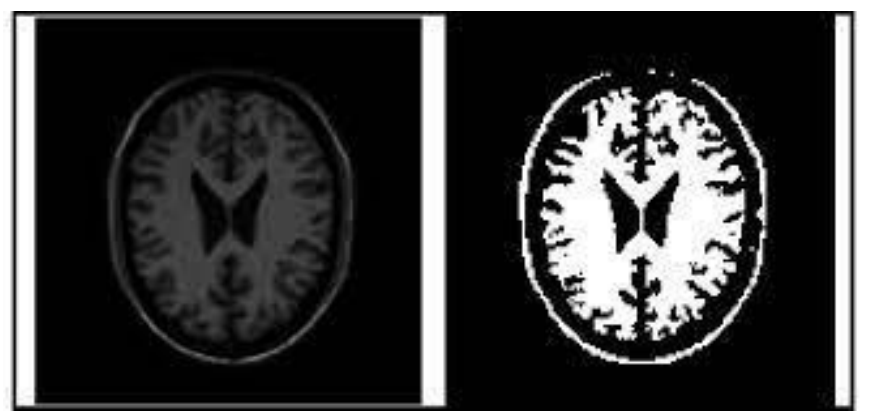

\section{Segmentation by Region based :}

In this technique pixels that are related to the same object are grouped for segmentation. The thresholding technique is bound with region based segmentation. The area that is detected for segmentation should be closed.
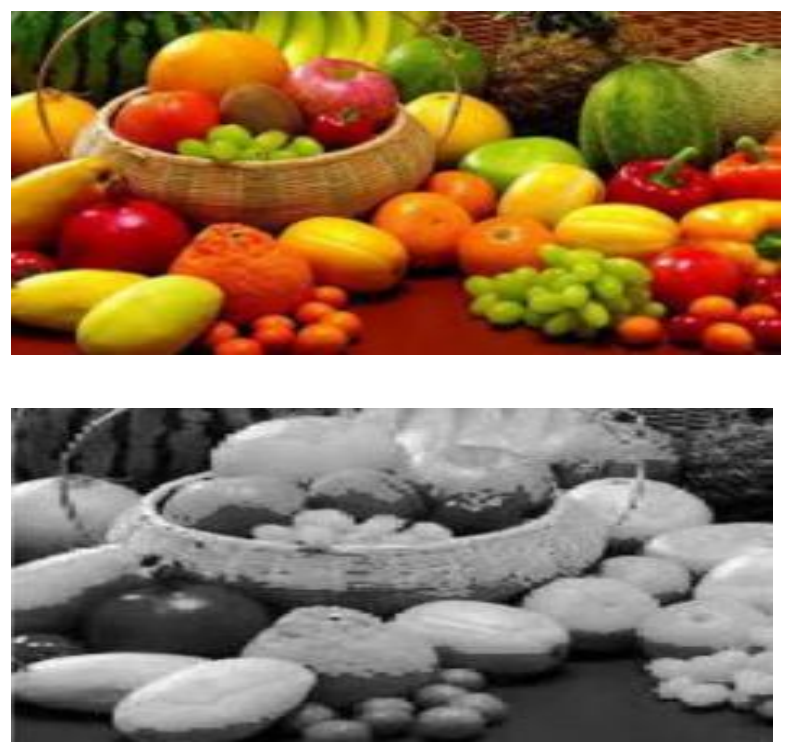

\section{Segmentation by Feature based Clustering:}

Clustering a process of organizing the groups based on its attributes. A cluster usually contains a group of similar pixels that belongs to a specific region and is different from other regions.
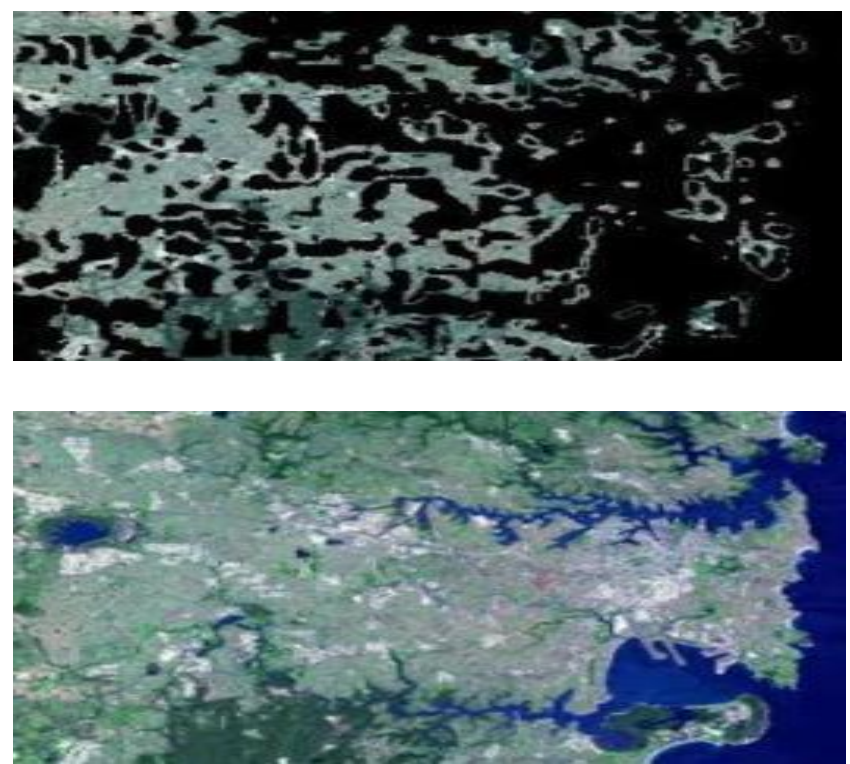


\section{Image Segmentation Applications:}

\section{Self-driving cars:ification:}

Image segmentation can be used in self-driving cars for giving easy distinctions between various objects. Be it traffic signals, signboards, humans, and cars. It can help the driving instruction algorithm to better assess the surroundings before generating the next instruction.

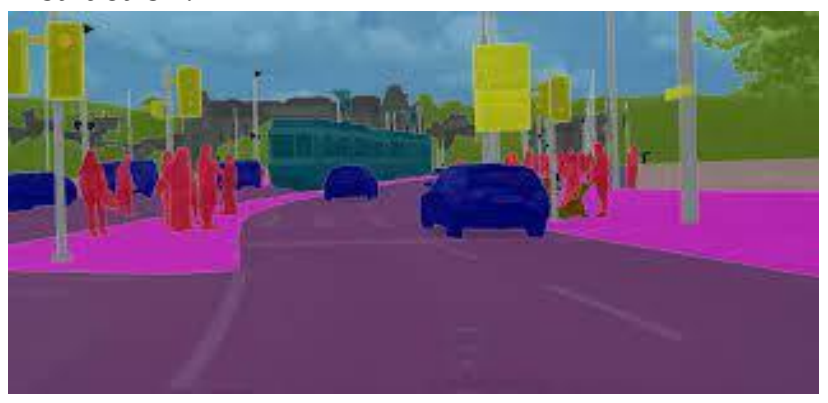

\section{Circuit Board Defect Detection}

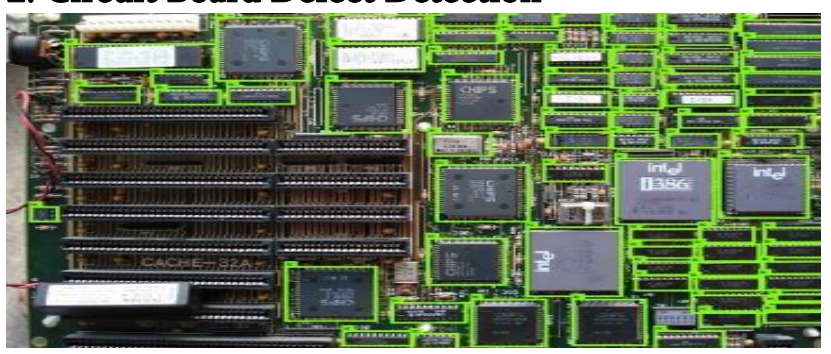

A company has to bear the responsibility of defective devices. If a camera backed with an Image Segmentation model keeps scanning for defects produced in the final product, a lot of money and time can be saved in fixing a defective device.

\section{Face detection:}

Nowadays, we have observed that the majority of cameras in phones support portrait mode. Portrait mode is technically an outcome of Image Segmentation. Apart from this, security surveillance will be much more effective when the faces are distinguishable from noisy objects.

\section{Medical Imaging:}

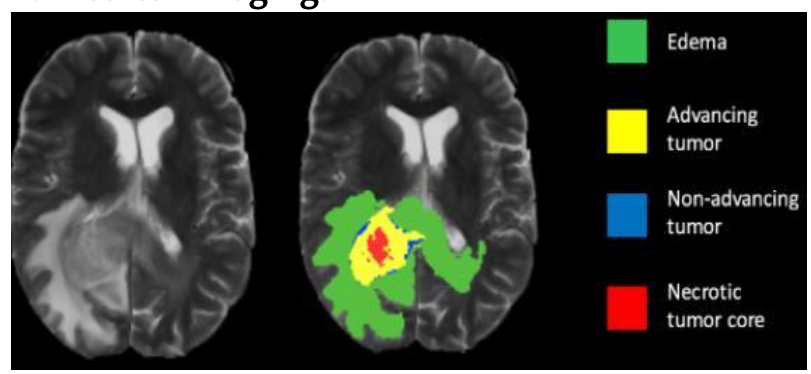

Image segmentation can be used to extract clinically relevant information from medical reports. For example, image segmentation can be used to segment tumors.

\section{Mask RCNN:}

How does Mask RCNN work? Mask RCNN uses an architecture similar to its predecessor Faster RCNN and also utilizes Fully convolution Network for pixel-wise segmentation. The Mask RCNN returns the binary object mask in addition to class label and object bounding box. Mask RCNN is good at pixel level segmentation.

1. Feature Extraction We utilize the ResNet 101 architecture to extract features from the input image. As a result, we get feature maps which are transmitted to Region Proposed Network

2. Region Proposed Network (RPN) After obtaining the feature maps, bounding box candidates are determined and thus RPN extracts RoI (Region of Interest)

3. RoI Pool Faster RCNN uses an RoI Pool layer to compute features from the obtained proposals in order to infer the class of the object and bounding box coordinates.

4. RoI Align RoI pool led to miss alignments in getting the Region of Interest due to quantization of RoI coordinates. Since pixel-level segmentation required specificity hence authors of the Faster RCNN cleverly solved it by implementing the RoI Align.

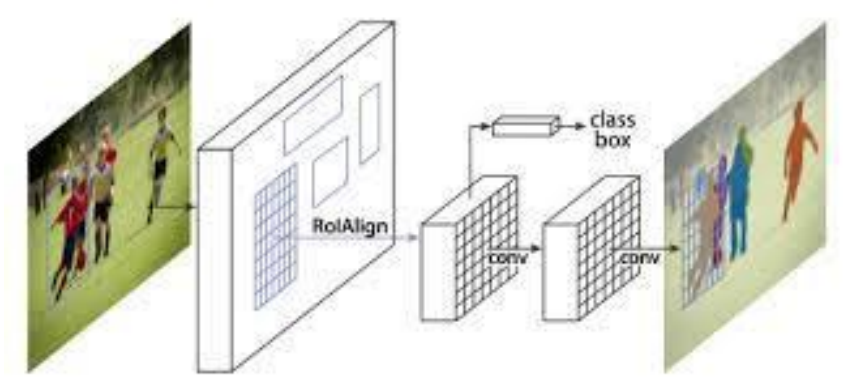




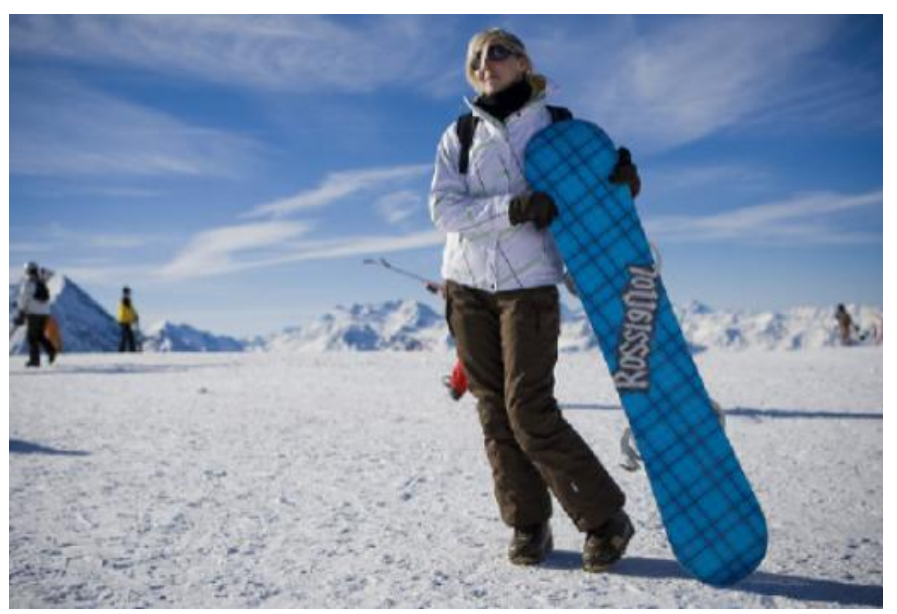

\section{CONCLUSION}

Image processing is the general issue in today's era, when we work with computer vision. It is in itself, a broad view to be considered. In order to process the image, we need to segment it so that it would become easier for the computer to understand. Image segmentation is the process of segmenting the image into various segments that could be used for further applications such as: Image understanding model, Robotics, Image analysis, Medical diagnosis, etc. Image segmentation is the process of partitioning an image into multiple segments, so as to change the representation of an image into something that is more meaningful and easier to analyze.

\section{REFERENCES}

[1]. Rajesh Dass,Priyanka,Swapna Devi,"Image Segmentation Techniques",IJECT Vol.3,Issue 1,JanMarch 2012.

[2]. Salem Saleh AI-amri,N.V Kalyankar,Khamitkar S.D,"Image Segmentation by using threshold Techniques",Journal of computing,Volume 2,issue 5,may 2010.

[3]. Sujata Saini and Komal Arora,"A Study on the Different Image Segmentation Techniques",International Journal of Information \& Computation Technology,ISSN 0974-2239 Volume 4,Number 14(2014),pp.1445-1452.
[4]. Arun Prakash Agrawali,Neha Tyagi,"Review on Digital Image Segmentation Techniques",ISSN-23945125 VOL 7,ISSUE 3,2020.

[5]. D.Kaur and Y.Kaur,"Various Image Segmentation Techniques:A

Review",Int.J.Compute.Sci.Mob.Compute,2014.

[6]. D.K.Raj,"A Review Paper:On Various Edge Detection Techniques",Int.J.Res.Appl.Sci.Eng.Technol,2017,doi: 10.22214/ijraset.2017.8074.

[7]. K.Jeevitha,A.Iyswariya,V.RamKumar,S.Mahaboob Basha,V.Praveen Kumar,"A Review on Various Segmentation Techniques in Image Processing",European Journal of Molecular \& Clinical Medicines ISSN 2515-8260 Volume 7,Issue 4,2020.

[8]. Steven L Eddins,Richard E.woods,Rafael C"Digital Image Processing"(2009)

[9]. Somesh Kumar Dewangan,Jyotir Moy Chatterjee,"A Study Report on Image Segmentation Techniques",International Journal of Advanced Computational Engineering and Networking,ISSN:2320-2106, Volume-5, Issue-11,Nov2017.

[10]. A.M.Khan,Ravi.S,"Image Segmentation Methods:A Comparative Study",International Journal of Soft Computing and Engineering(IJSCE),ISSN:22312307,Volume-3,Issue-4,September 2013.

[11]. R.C.Gonzalez and R.E.Woods."Digital Image Processing",third edition,PHI publication,2008.

[12]. Nikhil R Pal and Sankar K Pal,"A Review on Image Segmentation Techniques",Pattern Recognition,Vol 26,No.29,1993.

\section{Cite this article as :}

Raksha S Kale, Dr. Surabhi Thorat, "Image Segmentation Techniques with Machine Learning", International Journal of Scientific Research in Computer Science, Engineering and Information Technology (IJSRCSEIT), ISSN : 2456-3307, Volume 7 Issue 6, pp. 232-235, November-December 2021. Available at doi: https://doi.org/10.32628/CSEIT1217653 Journal URL : https://ijsrcseit.com/CSEIT1217653 